\title{
Herpetofauna del campus de la Universidad del Magdalena, Santa Marta, Colombia
}

\author{
Herpetofauna of the University of Magdalena, \\ Santa Marta, Colombia
}

\author{
Andrés Montes-Correa*, Juan David Jiménez-Bolaño*, Danny Vergara-Ríos*, \\ Yizeth Ávila-Silva*, Liliana Saboyá-Acosta*, Juan Manuel Renjifo*
}

Resumen

\begin{abstract}
Objetivos: Caracterizar la herpetofauna y determinar su distribución espacial en el campus de la Universidad del Magdalena. Metodología: El estudio se realizó mediante el método de búsquedas por encuentro casual empezando desde las 9:00-12:00, 16:00-18:00, 19:00-21:00 horas, en época seca y lluviosa. También se instalaron dos trampas de caída con una barrera de interceptación en la zona de bosque en recuperación para tratar de capturar el mayor número de especies posibles. Resultados: Entre abril de 2011 y abril de 2013 se registraron 39 especies de herpetos así, siete especies de anfibios y 32 especies de reptiles, distribuidas en tres y 14 familias respectivamente, en el campus de la Universidad del Magdalena, Santa Marta, Colombia. Conclusión: Los registros de estas especies documentan la importancia de los relictos de bosque seco para la conservación de poblaciones de anfibios y reptiles en la costa Caribe de Colombia, principalmente de este remanente dentro del casco urbano, por convertirse en islas de conservación en la ciudad de Santa Marta.
\end{abstract}

Palabras clave: Bosque seco tropical, Campus universitario, Herpetofauna, Riqueza de especies.

\begin{abstract}
Objectives: We characterize the herpetofauna and determine their spatial distribution at the campus of Magdalena University. Methodology: The study was conducted by the method of Visual encounter surveys starting from 9:00-12:00, 16:00 to 18:00, 19:00 to 21:00 hours, during dry and rainy season. We also installed two pitfall traps with an interception barrier in the recovering forest trying to capture as many species as possible. Results: Between April 2011 and April 2013 we recorded 39 species, seven species of amphibians and 32 species of reptiles distributed in three and 14 families respectively, on the campus of the University of Magdalena, Santa Marta, Colombia. Conclusions: The records of these species document the importance of dry forest remnants for the conservation of amphibians and reptiles populations on the Caribbean coast of Colombia, mainly of this remnant within the town, to become islands of conservation in the city of Santa Marta.
\end{abstract}

Keywords: Campus, Herpetofauna, Species richness, Tropical dry forest

\section{Introducción}

La región Caribe representa 11,6\% de la extensión de Colombia, ocupando el cuarto puesto en biodiversidad nacional (Salazar-Holguín et al.2009) y el quinto tanto de anfibios como reptiles (Cháves y Santamaría, 2006). Sin embargo la deforestación del bosque seco tropical (Bs-T) (formación vegetal más predominante de la región) es uno de los factores que puede generar cambios en los patrones de la diversidad y distribución de la herpetofauna.

El Bs-T ha sido considerado como uno de los ecosistema más degradados, fragmentado y menos conocido (Álvarez et al. 1997) y su cobertura no su-

Grupo de Investigación en Sistemática y Ecología de Anfibios y Reptiles, Programa de Biología, Universidad del Magdalena, Santa Marta,Colombia. e-mail: andresc.montes@gmail.com, ikakogitayrona@gmail.com, yizethas@gmail.com, herpetos4@gmail.com, lilianasaboya@gmail.com,juanmanuel.renjifo@gmail.com

Fecha recepción: Marzo 2, $2014 \quad$ Fecha aprobación: Noviembre 7, $2014 \quad$ Editor asociado: Mantilla H. 
pera $1.5 \%$ de lo que era originalmente (Etter 1993). La fragmentación avanzada que presenta esta formación vegetal afecta drásticamente a la herpetofauna, pues genera reducción de área disponible, decrecimiento de la calidad del hábitat y una mayor vulnerabilidad debido a las acciones antrópicas (Carvajal-Cogollo y Urbina-Cardona 2008); además, las poblaciones de anfibios y reptiles pueden ser muy sensibles al cambio del entorno donde viven porque son afectadas por barreras de dispersión creadas por la nueva matriz del ambiente (Block et al. 1998). Los estudios de estructura de comunidades de herpetofauna son importantes porque generan una aproximación al estado de conservación de las localidades y aportan información básica de los sitios, como es el caso de la región Caribe colombiana (BlancoTorres et al. 2013).

Las comunidades de anfibios y reptiles presentan grandes diferencias según la cobertura vegetal y la humedad que el hábitat les ofrece. Los anfibios son dependientes de la humedad para sobrevivir y reproducirse, mientras que los reptiles por su piel cubierta de escamas queratinizadas y su reproducción por medio del huevo amniota, presentan mayor independencia de esta variable ambiental (VargasSalinas y Bolaños 1999).

Los predios de la Universidad del Magdalena están en un lugar interesante y de fácil acceso para llevar a cabo estudios básicos de composición y estructura de las comunidades de anfibios y reptiles, por esta razón se realizó un inventario con el objetivo de conocer la composición de la herpetofauna y su distribución espacial en diferentes sectores del campus universitario.

\section{Metodología}

Área de estudio. El estudio se realizó en el campus de la Universidad del Magdalena, ciudad de Santa Marta, Magdalena, Colombia (11 ${ }^{\circ} 13^{\prime} 18,31^{\prime \prime} \mathrm{N}$, $74^{\circ} 11^{\prime} 08,80^{\prime \prime} \mathrm{W}$, elevación $\left.21 \mathrm{~m}\right)$. Esta localidad se encuentra ubicada en la zona de vida del Bosque Seco Tropical. El régimen de precipitación es de tipo unimodal-biestacional y un promedio anual de lluvia $608,8 \mathrm{~mm}$ anuales. El clima es semi-árido con marcado deficit hídrico en la época seca, con climas mega-termales (Rangel y Carvajal 2012)

El campus de la universidad (Figura 1) ocupa 30

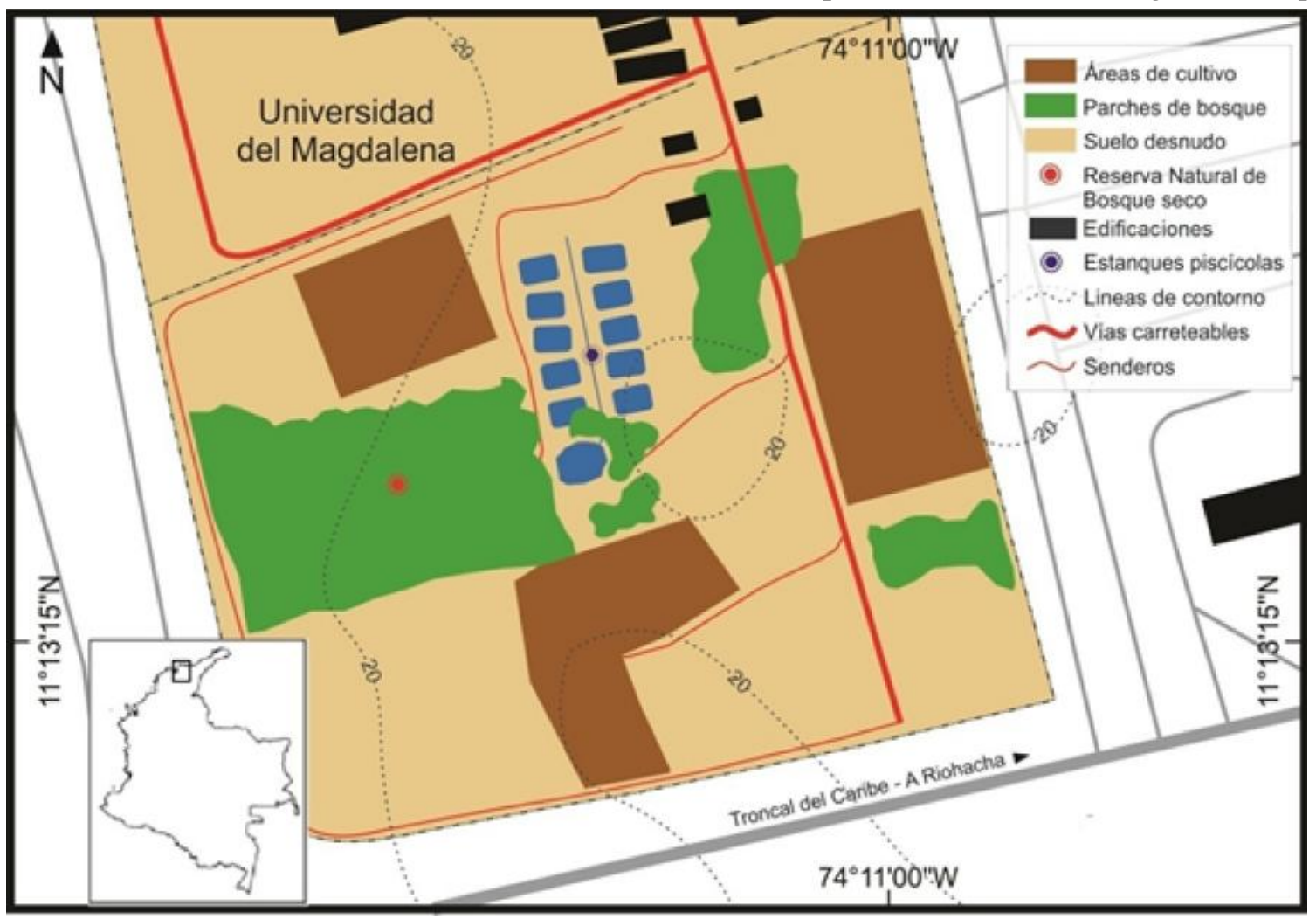

Figura 1. Mapa del área de estudio, haciendo énfasis en el Centro de Servicios Agropecuarios. 
ha, 10 de las cuales están dispuestas en edificaciones y un cuerpo de agua artificial y las 20 ha restantes hacen parte del Centro de Servicios Agropecuarios (CSA), el cual está ocupado en un $80 \%$ por rastrojos, pastizales, áreas de cultivo experimental, $10 \%$ por estanques artificiales y $5 \%$ es vegetación natural en recuperación conocida como Parcela de Bosque Seco tropical (área 50 x 200 m) (Strewe et al. 2009).

Los estudios botánicos adelantados en esta reserva, arrojan que la vegetación de este parche es secundaria, porque se trata de un bosque seco que ha pasado por distintos tipos de disturbios antropogénicos (tala, quema, etc.) y que está en progresiva recuperación y las familias Fabaceae, Boraginaceae y Capparidaceae son las más abundantes (Negritto com. pers.).

En el campus se diferencian por lo menos cinco áreas donde se pueden realizar avistamiento de anfibios y reptiles:

1. Zonas de cultivos ubicados en el CSA.

2. Rastrojos, en las zonas circundantes a la parcela permanente de bosque seco dentro del CSA.

3. Bosque seco en recuperación, ubicado en la parcela permanente del programa de Biología.

4. Zonas urbanas, correspondientes a todos los sectores de edificios, caminos, zonas verdes y canchas deportivas dentro del campus.

5. Cuerpos de aguas artificiales dentro del campus. Método de muestreo. Se realizó un censo visual sin restricciones (VES: Visual encounter sourvey) (Crump y Scott 1994) entre abril de 2011 y abril de 2013. Este consistió en realizar búsqueda activa en el CSA desde las 9:00-12:00, 16:00-18:00, y en la época lluviosa 19:00-21:00 horas. Dentro de la zona de bosque en recuperación se instalaron dos trampas de caída que consistían en dos baldes de un galón y una malla de angeo de dos metros de longitud entre ellos (Rodda y Dean-Bradley 2001) que se mantuvieron activas durante los recorridos y se revisaban cada dos días, las cuales permitieron la captura de especies de hábitos terrestres, fosoriales y semifosoriales. Los ejemplares capturados se llevaron a la colección de Herpetología de la Universidad del Magdalena para su identificación. Ningún ejemplar fue sacrificado para este estudio y todos fueron liberados después de ser identificados. Adicionalmente se realizó registro e identificación de especies de anuros a través de cantos. Se utilizaron las claves de Peters y Donoso-Barros (1970) y Peters y OrejasMiranda (1970) para reptiles y Cuentas et al. (2002) para anfibios. Además se realizó verificación de cantos con Acosta-Galvis (2014). Se realizaron registros ocasionales por especímenes llevados por personas de la comunidad universitaria a la colección o ejemplares atropellados en las vías dentro del campus.

Análisis de los datos. Por medio del el software «PRIMER 6» (versión 6.1.11) (Clarke y Gorley 2001) se utilizó el índice de Bray Curtis, con el objetivo de generar el dendrograma con el porcentaje de similitud entre zonas de avistamiento de la herpetofauna y así evaluar la homogeneidad del área de estudio. Se tiene en cuenta que si la similitud tiene un porcentaje mayor a $50 \%$ se considera un agrupamiento homogéneo. También se empleó este software para generar la curva de acumulación de especies.

\section{Resultados}

Los estimadores Jacknife 1 y 2 demuestran que el muestreo tuvo una representatividad oscilante entre $68,9 \%$ y $78,3 \%$. Al comparar la curva de especies de anfibios observadas con la de los estimadores, se evidencia que no hubo estabilización (Figura 2).

En total fueron registradas 39 especies; siete especies para anfibios representadas en las familias Leptodactylidae, Bufonidae e Hylidae y 31 especies de reptiles representadas en 15 familias (Tabla 1, Figura 3). Los anfibios estuvieron representados en su totalidad por el orden Anura, que representan 18\% de la herpetofauna total del área. Los reptiles capturados pertenecen a los órdenes Squamata y Testudines. Los lagartos (Squamata: Sauria) fueron los más diversos con 14 especies (37\%), seguido de las serpientes (Squamata: Serpentes) con 13 especies (34\%), mientras que las tortugas registradas solo componen $11 \%$ de la riqueza total de anfibios y reptiles.

El lugar con mayor número de especies de herpetos en el campus fue en la parcela de bosque seco en recuperación (Figura 4). Para el caso de anfibios, la zona de cuerpos de aguas artificiales registró la misma cantidad de especies que la del bosque en recuperación, en reptiles la segunda zona con mayor riqueza fue la de rastrojo. 


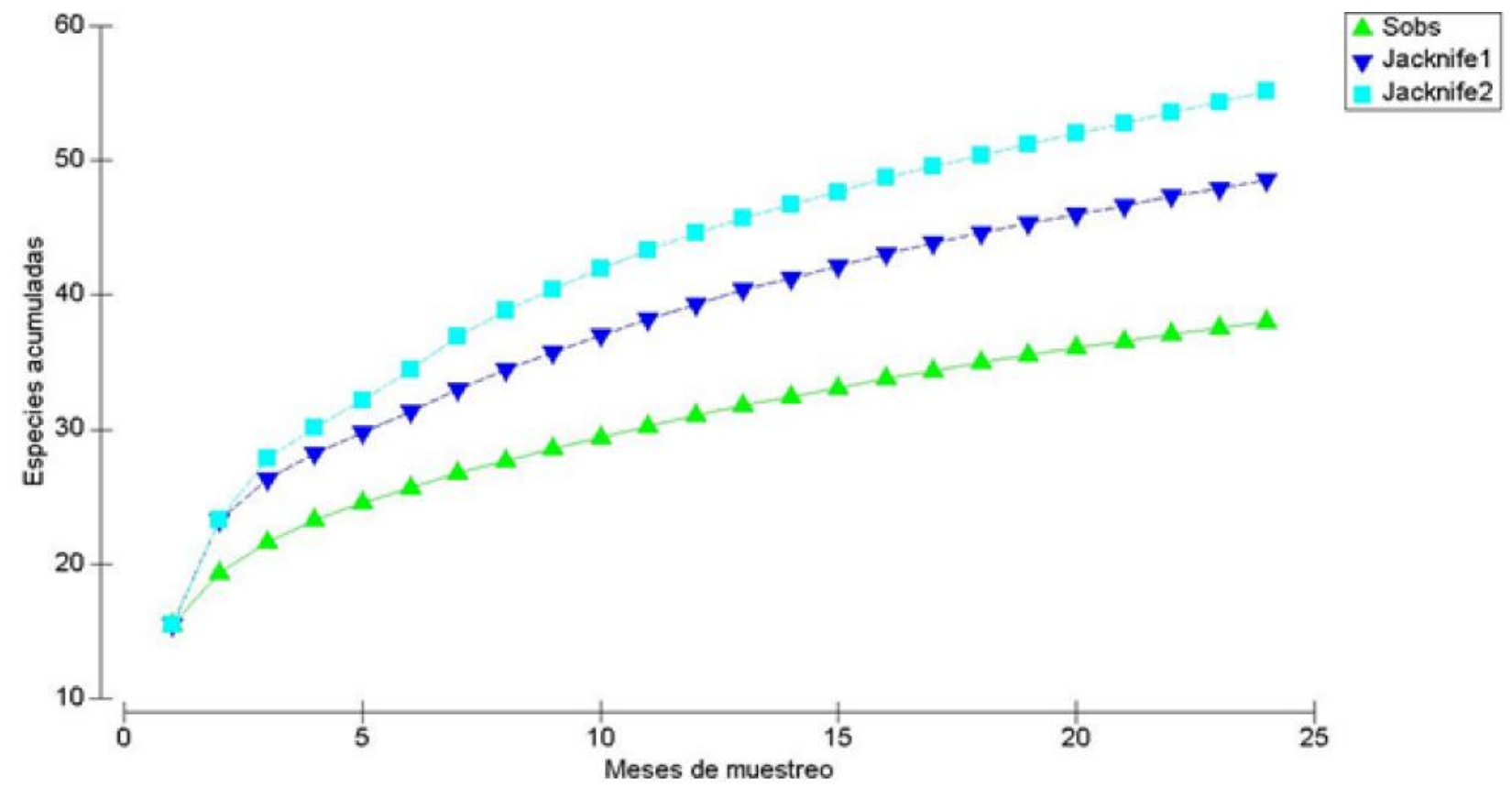

Figura 2. Curva de especies acumuladas durante los meses de muestreo.

El área de estudio mostró homogeneidad en la composición de especies (Figura 5), pero los cuerpos de aguas artificiales (CAA), al tener varias especies exclusivas debido a sus hábitos, presentó un porcentaje de similitud inferior a 50\% (32.2\% con respecto a las otras zonas de avistamiento). En el dendrograma se muestran otros dos agrupamientos, uno compuesto por bosque seco en recuperación (BSR) y cultivo (CUL) (con $68.2 \%$ de similitud), asimismo rastrojo (RAS) y zonas urbanas (ZU) son las zonas más símiles, con un porcentaje de $70 \%$.

\section{Discusión}

La riqueza de anfibios y reptiles para la zona de muestreo fue de 39 especies, 35 de ellas típicas de las formaciones vegetales secas de las tierras bajas del Caribe (Carvajal-Cogollo y Urbina Cardona 2008, Rueda-Solano y Castellanos 2010, Medina-Rangel 2011). En el caso de las tortugas acuáticas, todas son introducciones a los CAA del campus por motivos de ornato.

La mayoría de los herpetos reportados en este estudio corresponden a organismos adaptados a diversos tipos de ambientes, debido a sus hábitos generalistas y son muy comunes en áreas abiertas en las tierras bajas del Caribe colombiano. Por ejem- plo, Rhitella marina, R. humboldti y Leptodeira annulata estuvieron presentes en todas las zonas del campus. Estas especies presentan amplia tolerancia a hábitats perturbados (Zug y Zug 1979, Vitt 1996, Nabors 2005). En contraste, 18 especies estuvieron presentes en un solo sector del campus. Las especies del género Hemidactylus mostraron una marcada sinantropía, como lo documenta Caicedo-Portilla y Dulcey-Cala (2011), por ello solo se avistaron en las ZU. Con respecto a Lepidoblepharis sanctaemartae, como muchos pequeños geckos, se encuentra confinado a la hojarasca del bosque (Vitt et al.2005). Las tortugas de agua dulce se mantuvieron siempre en cercanías de los cuerpos de agua y Chelonoidis carbonarius se avistó tanto en el BSR y los CUL porque estos espacios le ofrecen frutas de las cuales se alimenta (Gallego-García et al. 2012).

Las serpientes Boa constrictor y Oxybelis aeneus se presentaron solo dentro del bosque. En otros estudios, B. constrictor también ha sido avistada en ambientes con mayor grado de perturbación (UrbinaCarona y Londoño 2003). En cuanto a la presencia exclusiva de $O$.aeneus en las formaciones vegetales arbustivas y arbóreas, va de acuerdo con la historia natural de la especie (Henderson 1974). Las demás especies de serpientes y el lagarto Bachia bicolor fueron registradas en una sola área del campus, de- 
Tabla 1. Listado de especies de anfibios y reptiles presentes en el campus de la Universidad y zona donde habitan.

\begin{tabular}{|c|c|c|c|c|c|}
\hline Taxón & CUL & RAS & BSR & ZU & CAA \\
\hline \multicolumn{6}{|l|}{ Clase Amphibia } \\
\hline \multicolumn{6}{|l|}{ Familia Bufonidae } \\
\hline Rhinella marina (Linnaeus 1758) & $\mathrm{x}$ & $\mathrm{x}$ & $\mathrm{x}$ & $\mathrm{x}$ & $\mathrm{X}$ \\
\hline Rhinella humboldti (Gallardo 1965) & $\mathrm{x}$ & $\mathrm{x}$ & $\mathrm{x}$ & $\mathrm{x}$ & $\mathrm{x}$ \\
\hline \multicolumn{6}{|l|}{ Familia Hylidae } \\
\hline Hypsiboas pugnax (Schmidt 1857) & & & $\mathrm{x}$ & & $x$ \\
\hline \multicolumn{6}{|l|}{ Familia Leptodactylidae } \\
\hline \multicolumn{2}{|l|}{ Leptodactylus fuscus (Schneider 1799) } & & $\mathrm{x}$ & & $\mathrm{x}$ \\
\hline \multicolumn{2}{|l|}{ Leptodactylus insularum (Barbour 1906) } & & $\mathrm{x}$ & $\mathrm{X}$ & $\mathrm{x}$ \\
\hline \multicolumn{2}{|l|}{ Engystomops pustulosus (Cope 1864) } & $\mathrm{x}$ & $\mathrm{x}$ & $\mathrm{x}$ & $\mathrm{x}$ \\
\hline \multicolumn{2}{|l|}{ Pleurodema brachyops (Cope 1869) } & $x$ & $x$ & $x$ & $x$ \\
\hline \multicolumn{6}{|l|}{ Clase Reptilia } \\
\hline \multicolumn{6}{|l|}{ Orden Squamata } \\
\hline \multicolumn{6}{|l|}{ Familia Teiidae } \\
\hline Ameiva praesignis (Baird y Girard 1852) & $\mathrm{X}$ & $\mathrm{X}$ & $\mathrm{x}$ & $\mathrm{x}$ & \\
\hline Ameiva bifrontata (Cope 1862) & $\mathrm{x}$ & $\mathrm{x}$ & $\mathrm{x}$ & $\mathrm{x}$ & \\
\hline Cnemidophorus lemniscatus (Linnaeus 1758) & $\mathrm{x}$ & $\mathrm{x}$ & $\mathrm{x}$ & $\mathrm{x}$ & \\
\hline \multicolumn{6}{|l|}{ Familia Gymnophthalmidae } \\
\hline \multicolumn{2}{|l|}{ Gymnophthalmus speciosus (Hallowell 1861) } & $\mathrm{x}$ & $\mathrm{X}$ & & \\
\hline Tretioscincus bifasciatus (Duméril 1851) & $\mathrm{X}$ & $\mathrm{x}$ & $\mathrm{x}$ & & \\
\hline \multirow{2}{*}{\multicolumn{6}{|c|}{ Bachia bicolor (Cope 1896) }} \\
\hline Familia Gekkonidae & & & & & \\
\hline \multicolumn{6}{|l|}{ Hemidactylus brookii (Gray 1845) } \\
\hline \multirow{2}{*}{\multicolumn{6}{|c|}{ Hemidactylus frenatus (Schlegel 1836) }} \\
\hline & & & & \\
\hline Lepidoblepharis sanctaemartae (Ruthven 1916) & & & $\mathrm{X}$ & & \\
\hline Gonatodes albogularis (Duméril y Bibron 1836) & $\mathrm{x}$ & $\mathrm{x}$ & $\mathrm{x}$ & $\mathrm{X}$ & \\
\hline \multicolumn{6}{|l|}{ Familia Phyllodactylidae } \\
\hline Phyllodactylus ventralis O'Shaughnessy 1875 & $\mathrm{x}$ & & $\mathrm{x}$ & & \\
\hline Thecadactylus rapicauda (Houttuyn 1782) & $\mathrm{x}$ & & & & \\
\hline \multicolumn{6}{|l|}{ Familia Dactyloidae } \\
\hline Anolis auratus Daudin 1802 & $x$ & $\mathrm{x}$ & $\mathrm{x}$ & & \\
\hline Familia Iguanidae & & & & & \\
\hline Iguana iguana (Linnaeus 1758) & $\mathrm{x}$ & $\mathrm{x}$ & $\mathrm{x}$ & $\mathrm{x}$ & \\
\hline Familia Boidae & & & & & \\
\hline Boa constrictor Linnaeus 1758 & $\mathrm{x}$ & & $\mathrm{X}$ & & \\
\hline Familia Anomalepididae & & & & & \\
\hline Liotyphlops albirostris (Peters 1857) & $x$ & $x$ & $x$ & $x$ & \\
\hline Familia Leptotyphlopidae & & & & & \\
\hline Epictia goudotii (Duméril y Bibron 1844) & & & & $\mathrm{x}$ & \\
\hline Familia Dipsadidae & & & & & \\
\hline Leptodeira septentrionalis Kennicott 1859 & & $x$ & & & \\
\hline Leptodeira annulata (Linnaeus 1758) & $\mathrm{x}$ & $\mathrm{x}$ & $\mathrm{x}$ & $\mathrm{x}$ & $\mathrm{x}$ \\
\hline Phimophis guianenesis (Troschel 1848) & & & & $\mathrm{X}$ & \\
\hline Enulius flavitorques (Cope 1868) & & $\mathrm{x}$ & & $x$ & \\
\hline Familia Colubridae & & & & & \\
\hline Mastigodryas boddaertii (Sentzen 1796) & & $\mathrm{x}$ & & & \\
\hline Oxybelis aeneus (Wagler 1824) & & & $\mathrm{x}$ & & \\
\hline Tantilla semicincta (Duméril, Bibron y Duméril 18 & 854) & $\mathrm{x}$ & $\mathrm{x}$ & & $\mathrm{x}$ \\
\hline Tantilla melanocephala (Linnaeus 1758) & $\mathrm{x}$ & & $x$ & & \\
\hline
\end{tabular}


Tabla 1. Listado de especies de anfibios y reptiles presentes en el campus de la Universidad y zona donde habitan (continuación).

\begin{tabular}{|c|c|c|c|c|c|}
\hline Taxón & CUL & RAS & BSR & ZU & CAA \\
\hline \multicolumn{6}{|l|}{ Familia Viperidae } \\
\hline Crotalus durissus Linnaeus 1758 & & $\mathrm{X}$ & $\mathrm{X}$ & & \\
\hline Porthidium lansbergii (Schlegel 1841) & & & $x$ & $\mathrm{X}$ & \\
\hline Familia Elapidae & & & & & \\
\hline Micrurus dissoleucus (Cope 1860) & & $x$ & $x$ & $x$ & \\
\hline Orden Testudines & & & & & \\
\hline \multicolumn{6}{|l|}{ Familia Podocnemididae } \\
\hline Podocnemis lewyana (Duméril 1852) & & & & & $\mathrm{X}$ \\
\hline \multicolumn{6}{|l|}{ Familia Kinosternidae } \\
\hline Kinosternon scorpioides (Linnaeus 1766) & & & & & $x$ \\
\hline \multicolumn{6}{|l|}{ Familia Testudinidae } \\
\hline Chelonoidis carbonaria (Spix 1824) & $X$ & & $x$ & & \\
\hline \multicolumn{6}{|l|}{ Familia Emydidae } \\
\hline Trachemys callirostris (Gray 1855) & & & & & $\mathrm{X}$ \\
\hline
\end{tabular}

CUL: cultivo; RAS: rastrojo; BSR: bosque seco en recuperación; CAA: cuerpos de aguas artificiales; ZU: Zonas urbanas.

bido a que sus hábitos crípticos dificultaron su observación.

Del total de especies encontradas en el campus, 13 son consideradas colonizadoras comunes de áreas intervenidas (R. marina, R. humboldti, Hypsiboas pugnax, Tretrioscincus bifasciatus, Gonatodes albogularis, Thecadactylus rapicauda, Iguana iguana, Ameiva praesignis, Cnemidophorus lemniscatus, Hemidactylus frenatus, $H$. brookii, Anolis auratus) (Dueñez-Gómez et al. 2004, Medina-Rangel 2011).

La mayoría de los registros de reptiles y anfibios fueron dentro de la parcela experimental de bosque seco en recuperación; este hecho puede estar asociado con el restablecimiento de los servicios ecológicos en este parche de bosque como la regulación del balance hídrico, el mantenimiento de la fertilidad y estabilidad física de los suelos y la generación de hábitats apropiados para la herpetofauna (Cavelier 1998, Kattan 1998, Vieria y Scariot 2006). Tanto reptiles como anfibios presentan una relación directa con la temperatura y balance hídrico lo cual limita su distribución en un área. Factores como la cobertura vegetal, permiten en gran manera el desarrollo de este tipo de organismos ya que los microclimas formados entre los microhábitat son los que determinan la presencia o ausencia de ciertos grupos.

El desplazamiento de las especies a lo largo de su hábitat está sujeto en su mayoría a la búsqueda de lugares con mayor disponibilidad de luz solar directa, sin embargo es importante resaltar que la mayo- ría de lagartos habitan donde la radiación solar es reducida por nubosidades o por la cobertura del dosel (Nicholson et al. 2005, Catenazzi et al. 2005). Esto explica la mayor presencia de lagartos en zonas con cobertura vegetal más densa, como el bosque y los cultivos. Las serpientes también mostraron este comportamiento (Figura 6). Los ofidios venenosos que habitan el campus están en capacidad de tolerar ambientes con diferente grado de perturbación (Rueda-Almonacid et al. 2008, Galvis-Peñuela et al. 2011), y se les ha encontrado muy cerca de edificaciones, atropelladas en las vías y parqueaderos o forrajeando de noche en lugares con abundante flujo de personas.

Del total de anfibios registrados para el Caribe y la zona de Santa Marta, solo se encontró un porcentaje bajo para el campus de la Universidad. La baja diversidad registrada pude explicarse por el limitante hídrico que este grupo presenta. Los anfibios de zonas secas tienen que desarrollar estrategias que le permitan sobrevivir ante los largos períodos de sequía (Cuentas et al. 2002), como es el cambio en el patrón de actividad, habilidad para la captación de agua y desarrollo de amplios rangos de tolerancia a la deshidratación; algunas especies por ejemplo, pueden tolerar la deshidratación hasta un 50\% del total de su masa corporal y $60 \%$ del total de contenido de agua (Thorson 1955).

Históricamente, la Universidad del Magdalena hacía parte de una red de pequeños parches de bos- 


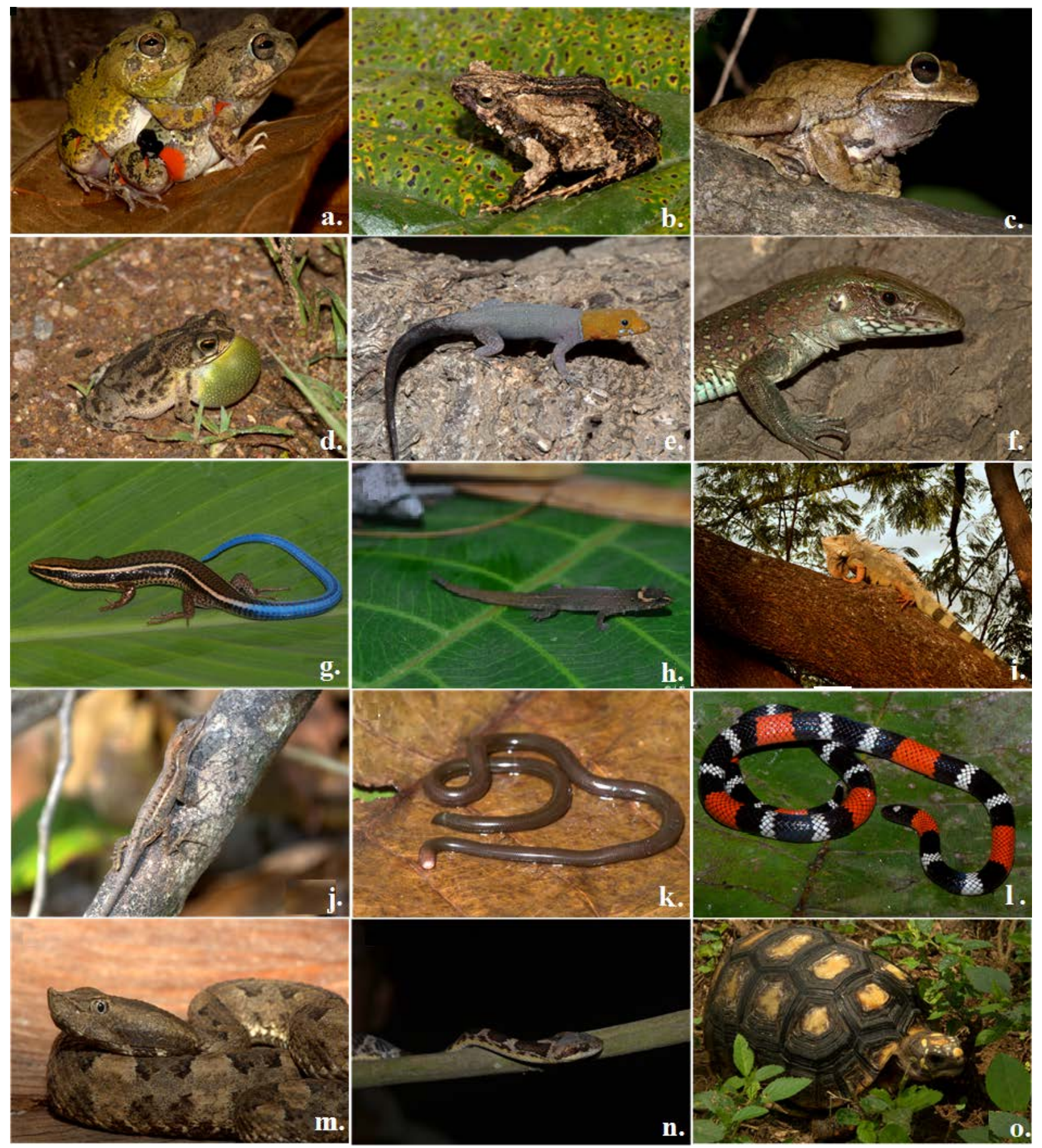

Figura 3. Herpetofauna conspicua del campus de la Universidad del Magdalena. a. Pleurodema brachyops, b. Engystomops pustulosus, c. Hypsiboas pugnax, d. Rhinella humboldti, e. Gonatodes albogularis, f. Ameiva praesignis, g. Tretioscincus bifasciatus, h. Lepidoblepharis sanctaemartae, i. Iguana iguana, j. Anolis auratus, k. Liotyphlops albirostris, I. Micrurus dissoleucus, m. Porthidium lansbergii, n. Leptodeira annulata, o. Chelonoidis carbonarius.

Fotos: LP Saboyá-Acosta (h, i, j, n) y JM Renjifo (a, b, c, d, e, f, g, k, I, m, o).

que dispersos en el área marginal de la parte baja del río Manzanares, extendidos desde el sector de
Mamatoco hasta el Minuto de Dios. Los residentes más viejos de la zona aseguran la existencia de cuer- 


\section{a. anfibios}

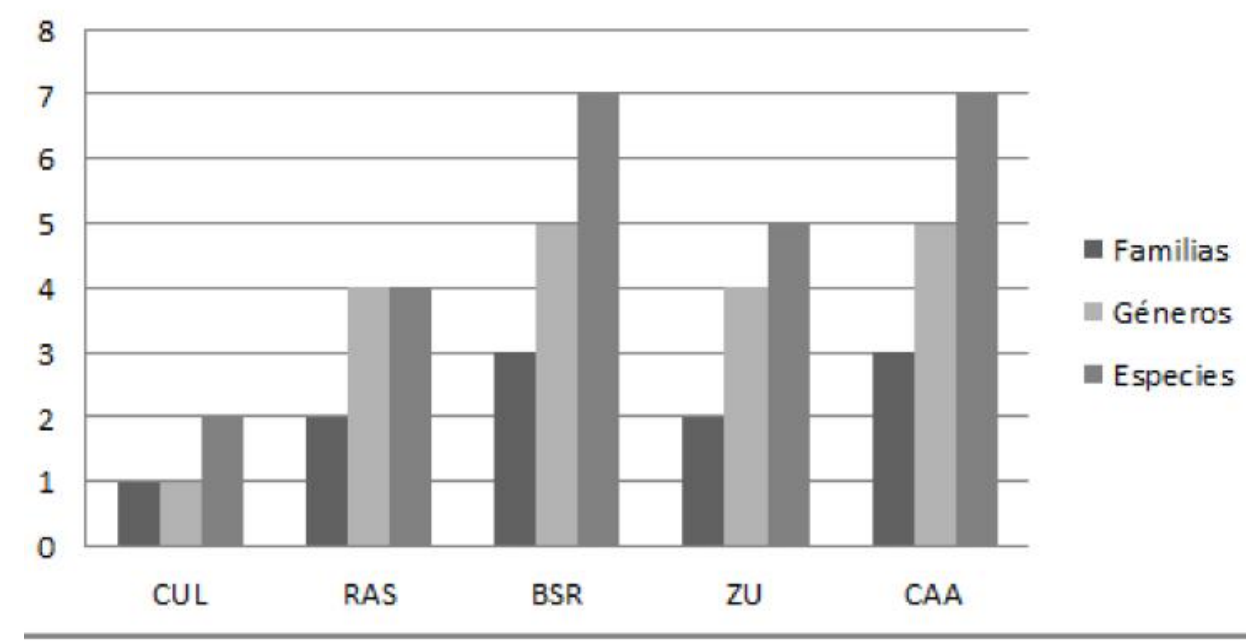

b. reptiles

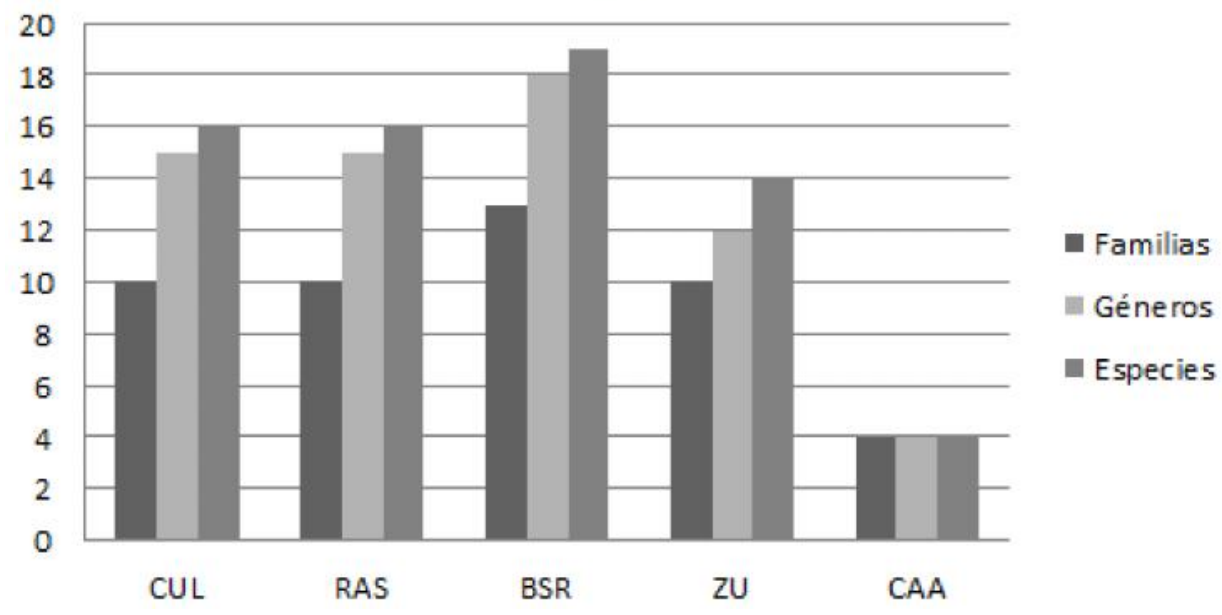

Figura 4. Diversidad de familias, géneros y especies de anfibios y reptiles según la zona de avistamiento en el campus.

pos de agua asociados con este río. Sin embargo, el crecimiento del casco urbano y la creación de barreras físicas alrededor de la Universidad, aislaron la fauna presente en el campus. A pesar de los daños que la fragmentación causa, los remanentes de bosques constituyen importantes refugios y reservorios de fauna, llegando a tener una mayor diversidad biológica que su matriz circundante (Cavelier 1998, Vieria y Scariot 2006).

\section{Agradecimientos}

Le agradecemos al Profesor Jeiner Castellanos
Barliza, pues junto a él surgió nuestra pregunta de investigación. A los profesores Willinton Barranco, Germán Galvis Vergara, León Pérez Carmona, María Negritto y Paulo Tigreros por los aportes materiales e intelectuales. Agradecemos a Andrés Tette, Luis Batista, Jorge Gómez y Daniela Maiguel del Risco por su colaboración con el trabajo de campo, al compañero y amigo Carlos Villa de León por su ayuda en la construcción del mapa y por último al doctor Ralf Strewe, pues sin su esfuerzo por conservar la actual parcela de bosque seco, no hubiéramos podido realizar este trabajo. 


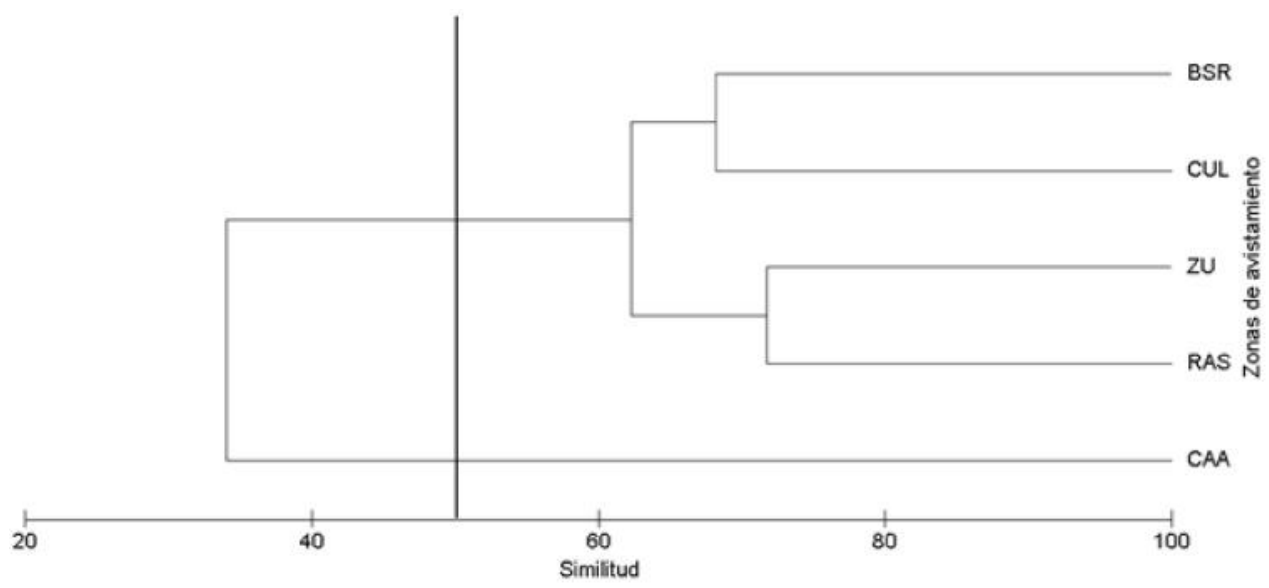

Figura 5. Similitud entre las zonas de avistamiento de herpetofauna del campus de la Universidad del Magdalena.

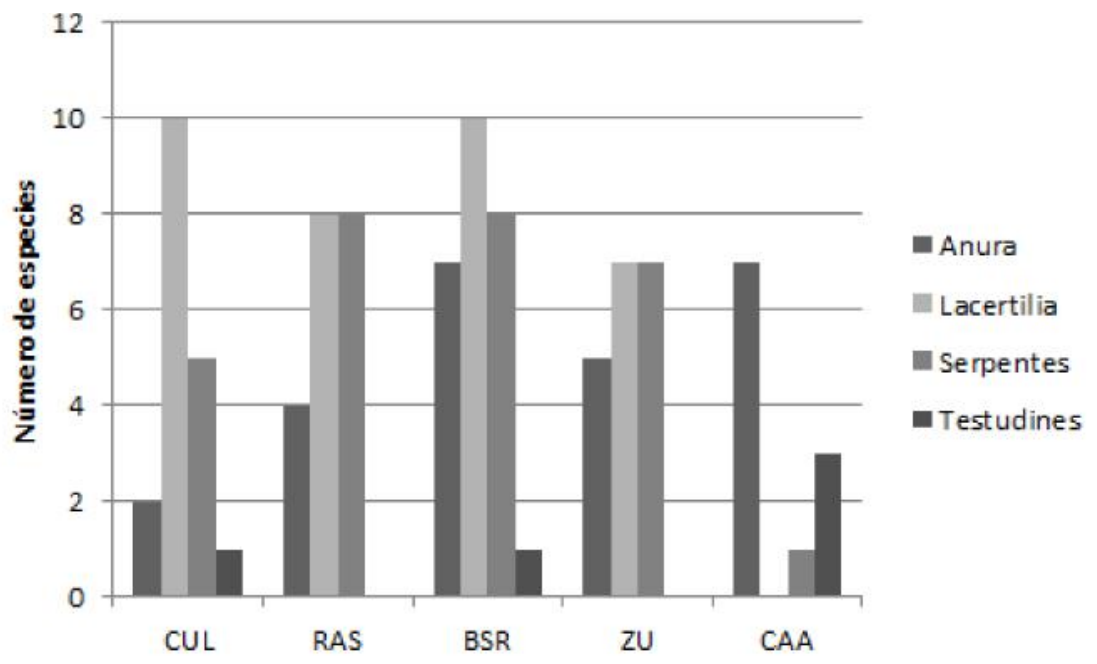

Figura 6. Número de especies de herpetos según la zona de avistamiento.

\section{Literatura citada}

Acosta-Galvis AR. 2014. Lista de los Anfibios de Colombia. V.02.2014.0. (En línea) 2014 (Acceso 20 de marzo). URL disponible en: www.batrachia.com

Álvarez MF, Escobar F, Gast F, Mendoza H, Repizzo A, Villareal H. 1997. Bosque seco tropical. En: Informe nacional sobre el estado de la biodiversidad colombiana. Bogotá: Instituto de Investigación en Recursos Biológicos Alexander von Humboldt; pp. 56-75.

Blanco-Torres A, Báez L, Patiño-Flores E, Renjifo JM. 2013. Herpetofauna del valle medio del río Ranchería, la Guajira, Colombia. Rev Biodivers Neotrop. 3 (2): 11322.

Block WM, Morrinson ML, Scott PE. 1998. Development and evaluation habitat models for Herpetofauna and small mammals. Forest Sci. 44 (3): 430-7.

Caicedo-Portilla R, Dulcey-Cala CJ. 2011. Distribución del gecko introducido Hemidactylus frenatus (Dumeril y
Bribon 1836) (Squamata: Gekkonidae) en Colombia. Biota Colomb. 12 (2): 45-56.

Carvajal-Cogollo JE, Urbina-Cardona JN. 2008. Patrones de diversidad y composición de reptiles en fragmentos de bosque seco tropical en Córdoba, Colombia. Trop Conserv Sci. 1 (4): 397-416.

Catenazzi A, Carrillo J, Donnelly MA. 2005. Seasonal and geographic eurythermy in a coastal Peruvian lizard. Copeia. 2005 (4): 713-23.

Cavelier J. 1998. Restauración de elementos de biodiversidad: Definición de restauración ecológica. En: Informe nacional sobre el estado de la biodiversidad. Bogotá: Instituto de Investigación en Recursos Biológicos Alexander von Humboldt; pp. 56-75.

Cháves ME, Santamaría M (eds.). 2006. Informe Nacional sobre el Avance en el Conocimiento y la Información de la Biodiversidad 1998-2004. Tomo 2. Bogotá: Instituto de Investigación en Recursos Biológicos Alexander von Humboldt; p. 33. 
Clarke KR, Gorley RN. 2001.PRIMER user manual: Plymouth routines in multivariate ecological research. Plymouth: Plymouth Marine Laboratory; $91 \mathrm{p}$.

Crosswhite DL, Fox SF, Thill RE. 1999. Comparison of methods for monitoring reptiles and amphibians in upland forests of the Ouachita Mountains. Proc Okla Acad Sci. 79: 4550 .

Crump ML, Scott NG. 1994. Standard techniques for inventory and monitoring. En: Heyer MA, Donelly RW, McDiarmid LA, Hayek C. Foster MS (ed.). Measuring and monitoring biological. Standard methods for amphibians. Washington DC: Smithsonian Institution Press; pp. 77171.

Cuentas D, Borja R, Lynch JD, Renjifo JM. 2002. Anuros del departamento del Atlántico y Norte de Bolívar. Barranquilla: Cencys 21; 117 p.

Dueñez-Gómez F, Muñoz-Guerrero J, Ramírez-Pinilla MP. 2004. Herpetofauna del corregimiento Botillero (El Banco, Magdalena) en la depresión Momposina de la región Caribe Colombiana. Actual Biol. 26 (81): 65-74.

Etter A. 1993. Diversidad ecosistémica en Colombia hoy. En: Cárdenas S y Correa HD. Nuestra diversidad biótica. Bogotá: CEREC y Fundación Alejandro Ángel Escobar; pp. 43-61.

Gallego-García N, Cárdenas-Arévalo G, Castaño-Mora OV. 2012. Chelonoidis carbonaria (Spix 1824). En: Páez VP, Morales-Betancourt MA, Lasso CA, Castaño-Mora OV, Bock B. V. Biología y conservación de las tortugas continentales de Colombia. Bogotá: Serie de recursos hidrobiológicos y pesqueros continentales de Colombia. pp. 406-411.

Galvis-Peñuela PA, Mejía-Tobón A, Rueda-Almonacid JV. 2011. Fauna silvestre de la Reserva Forestal Protectora Montes de Oca, La Guajira. Riohacha: Corpoguajira; 822 pp.

Henderson RW. 1974. Aspects of the ecology of the Neotropical vine snake, Oxybelis aeneus (Wagler). Herpetologica. 30 (1): 19-24.

Kattan G. 1998. Transformación del paisaje y fragmentación del hábitat. En: Informe Nacional sobre el estado de la biodiversidad. Bogotá: Instituto de Investigación en Recursos Biológicos Alexander von Humboldt; pp. 5675.

Medina-Rangel GF. 2011. Diversidad alfa y beta de la comunidad de reptiles en el complejo cenagoso de Zapatosa, Colombia. Rev Biol Trop. 51 (2): 935-68.

Nabors LK. 2005. Amphibians and human-altered habitats: A case study in the llanos of Venezuela. (PhD thesis). Davis: University of California.

Nicholson KL, Torrence SM, Ghioca DM, Bhattacharjee J, Andrei AE, Owen J, et al. 2005. The influence of temperature and humidity on activity patterns of the lizards Anolis stratulus and Ameiva exsul in the British Virgin Islands. Caribb J Sci. 41 (4): 870-3.

Peters JA, Donoso-Barros R. 1970. Catalogue of Neotropical Squamata: part II. Lizards and Amphisbaenians. Washington DC: Smithsonian Institution United States National Museum; 293 p.
Peters JA, Orejas-Miranda B.1970. Catalogue of Neotropical Squamata: part I. Snakes. Washington DC: Smithsonian Institution United States National Museum; 347 p.

Rangel JO, Carvajal-Cogollo JE. 2012. Clima de la región Caribe colombiana. En: Colombia Diversidad Biótica XII: La región Caribe de Colombia. Bogotá: Instituto de Ciencia Naturales, Universidad Nacional de Colombia; pp. 67-129.

Rodda GH, Dean-Bradley K. 2001. Excess density compensation of island herpetofaunal assemblages. J Biogeogr. 29: 623-32.

Rueda-Almonacid JV, Velásquez AA, Galvis-Peñuela PA, Gualdrón-Duarte J. 2008. Reptiles. En: RodríguezMahecha JV, Rueda-Almonacid JV, Gutiérrez-Hinojosa TD (eds.). Guía ilustrada de fauna del Santuario de Vida Silvestre Los Besotes, Valledupar, Cesar, Colombia. Serie de guías tropicales de campo $\mathrm{N}^{\mathrm{o}} 7$. Conservación Internacional-Colombia. Bogotá: Editorial Panamericana, Formas e Impresos; 574 p.

Rueda-Solano LA, Castellanos-Barliza J. 2010. Herpetofauna de Neguanje, Parque Nacional Natural Tayrona, Caribe Colombiano. Acta Biol Colomb. 15 (1): 195-206.

Salazar-Holguín F, Benavides-Molineros J, TrespalaciosGonzález OL, Pinzón-Flórez LF. 2009. Informe sobre el Estado de los Recursos Naturales Renovables y del Ambiente 2009. Tomo III Perfil. Bogotá: IDEAM, SIAC.

Strewe R, Villa-de León C, Alzate J, Beltrán J, Moya J, Navarro C, Útria G. 2009. Las aves del campus de la Universidad del Magdalena, Santa Marta, Colombia. Intropica. 4: 7789.

Thorson TB. 1955. The relationship of water economy to terrestrialism in amphibians. Ecology. 36: 100-16

Urbina-Cardona JN, Londoño MC. 2003. Distribución de la comunidad de herpetofauna asociada a cuatro áreas con diferente grado de perturbación en la Isla Gorgona, Pacífico colombiano. Rev Acad Colomb Cienc Exact Fis Nat. 27 (102): 105-13.

Vargas-Salinas F, Bolaños ME. 1999. Anfibios y reptiles presentes en hábitats perturbados de selva lluviosa tropical en el Bajo Anchicayá, Pacífico colombiano. Rev Acad Colomb Cienc Exact Fis Nat. 23 (supl especial): 499511.

Viera DL y Scariot A. 2006. Principles of natural regeneration of tropical dry forest for restoration. Restoration Ecol. 14 (1): 11-20.

Vitt LJ. 1996. Ecological observations on the tropical colubrid snake Leptodeira annulata. Herpetol Nat Hist. 4 (1): 6976.

Vitt LJ, Sartorius SS, Avila-Pires TCS, Zani PA, Espósito MC. 2005. Small in a big world: ecology of leaf-litter geckos in new world tropical forests. Herpetol Monogr. 19: 13752.

Zug G, Zug P. 1979. The marine toad, Bufo marinus: a natural history resume of native populations. Smithsonian Contributions to Zoology. $\mathrm{N}^{\mathrm{o}} 284$. Washington, DC: Smithsonian Institution Press; 58 pp. 